\title{
A Fatigue Crack Length Estimation and Prediction using Trans- fitting with Support Vector Regression
}

\author{
Myeongbaek Youn ${ }^{1}$, Yunhan Kim², Dongki Lee ${ }^{3}$, and Minki Cho ${ }^{4}$ \\ ${ }^{1,2}$ Department of Mechanical and Aerospace Engineering, Seoul National University, Seoul, 08826, Republic of Korea \\ mbyun1420@snu.ac.kr \\ gav0304@snu.ac.kr \\ ${ }^{3,4}$ Material \& Production engineering Research Institute, LG Electronics Inc., Gyeonggi-do 17709, Republic of Korea \\ dongki2.lee@lge.com \\ minki.cho@lge.com
}

\begin{abstract}
This paper explains the proposed method of Team SeouLG in detail for crack length estimation and prediction based on wave signals. The proposed method consists of two parts; (1) crack length estimation using support vector regression (SVR), (2) crack length prediction using new trans-fitting method. For the estimation of the crack length, we define the features based on the filtered wave signals and construct the model using SVR method. The hyper-parameters of the SVR model are selected based on grid search algorithm. The prediction of the crack length is based on the previous crack length, which is estimated based on the wave signals. In this part, we apply a new proposed trans-fitting method. The trans-fitting method updates the selected candidate function to fit the trend of the crack propagation from the training dataset. By translocating the selected candidate functions to the estimated crack length, we can predict the crack length of the target cycles. The proposed method is validated with the new given specimens. The results show that the proposed method can estimate and predict the crack length to lead Team SeouLG to the first place in 2019 PHM Conference Data Challenge.
\end{abstract}

